\title{
OPTIMAL ESTIMATION OF DISTRIBUTED SCATTERER PHASE HISTORY PARAMETERS FROM METER-RESOLUTION SAR DATA
}

\author{
Yuanyuan Wang ${ }^{(1)}$, Xiao Xiang Zhu ${ }^{(1)}$, Richard Bamler ${ }^{(1,2)}$ \\ (1) Lehrstuhl für Methodik der Fernerkundung, \\ Technische Universität München, \\ Arcisstraße 21, 80333 Munich, Germany \\ Email: wang@bv.tum.com \\ (2) Remote Sensing Technology Institute (IMF), \\ German Aerospace Center (DLR), \\ Oberpfaffenhofen, \\ D-82234 Weßling, Germany
}

\begin{abstract}
Measuring the long-term line-of-sight deformation using a multipass SAR data stack by standard persistent scatterer technique has been explored since the late 1990s. Researches have been continuously conducted on increasing the data coverage at non-PSrich areas. The recently developed SqueeSAR ${ }^{\mathrm{TM}}$ technique has validated the potential of extracting useful information from distributed scatterers. With the availability of high resolution TerraSAR-X spotlight data, this technique can benefit greatly from its higher data density and quality.
\end{abstract}

This article presents an algorithm of parameter estimation at distributed scatterers by maximum likelihood estimator in high resolution TS-X spotlight data. Different to SqueeSAR ${ }^{\mathrm{TM}}$, this article pays particular attention to the accurate covariance matrix estimation for phase history retrieval on each individual distributed scatterer. Solutions are presented for adaptive sample selection by a different statistical test (Anderson-Darling). An adaptive multiresolution defringe algorithm is introduced to cope with the problem of accurate fringe removal and in turn accurate covariance matrix estimation. And finally maximum likelihood estimator was employed to estimate the model parameters by weighting each measurement according to its coherence. By combining both the persistent scatterers and distributed scatterers, the increase in the capable monitoring area is phenomenal.

Index Terms - Interferometric SAR, distributed scatterers, covariance matrix, phase history

\section{INTRODUCTION}

The new generation of space-borne SAR systems, like TerraSAR$\mathrm{X}$ (TS-X), provides higher resolution, shorter revisit time, and better orbit control, which opens a new attitude to look at the distributed scatterers (DS), and enables the usage of them. The absence of such systems before concedes the research to mainly focus on persistent scatterer (PS). The standard PS technique is essentially a model fitting problem by maximizing the unweighted sum of the residual phase between the model and the measurements (ensemble coherence maximization [1]). PS ensures the quality of the measurements, and therefore weighting is not absolutely necessary.

In the case of DS, they are assumed to be independent circular Gaussian random variates [2]. Maximum Likelihood Estimator (MLE) was developed to estimate the interferogram phase [3]. But problem arises at the reliability of the covariance matrix due to the longer revisit time in the old time SAR system causing strong decorrelation of the target. Most of the studies were presented with simulated data. Subsequent research has been done on the decorrelation models and improvement on the MLE [4]. The recently developed SqueeSAR ${ }^{\mathrm{TM}}$ technique [5] presented a method to extract information from the DS by making use of an adaptive sample selection strategy which applies a statistical test on each pixel to correctly select samples and accurately estimate the covariance matrix. Specific research was then started on the characteristics of different statistical tests [6].

\section{MAXIMUM LIKELIHOOD ESTIMATOR}

According to [4], the likelihood function to be maximized is expressed as follows:

$$
\{\hat{h}, \hat{v}\}=\arg \max \left\{\frac{\exp \left(-\mathbf{i}^{H} \Phi(h, v) \mathbf{C}_{0}^{-1} \Phi(h, v)^{H} \mathbf{i}\right)}{\operatorname{det}\left|\mathbf{C}_{0}\right| \pi^{\mathrm{N}}}\right\}
$$

where

i: $\quad$ vector of measurements

Ф: $\quad$ diagonal matrix containing the phase of the model

$\mathbf{C}_{0}$ : $\quad$ absolute value of the covariance matrix

$h, v: \quad$ digital elevation model (DEM) error, line-of-sight (LOS)

$\mathrm{N}$. displacement velocity

number of images

The phase of the covariance matrix resembles the interferogram of all the possible combinations of any two acquisitions. And its absolute values represent the correlation between any two acquisitions. The $\mathbf{C}_{0}^{-1}$ matrix in the MLE applies weighting on each measurement. Therefore, the correctness of the covariance plays an important role on the MLE.

\section{COVARIANCE MATRIX ESTIMATION}

The covariance between two acquisitions $n$ and $k$ is estimated by taking the mean value of $\mathrm{M}$ nearby samples.

$$
\hat{c}_{n, k}=\frac{1}{\mathrm{M}} \sum_{m} i_{n}(m) i_{k}^{*}(m)
$$

According to Reed-Mallett-Brennan detection loss theory, the number $\mathrm{M}$ is usually large than $2 \mathrm{~N}$, in order to maintain a loss of the likelihood (shown in Eq. (1)) of less than 3dB. The loss of the likelihood is concluded in [7] as: 


$$
\text { loss }=-10 \log _{10}[(\mathrm{M}+2-\mathrm{N}) /(\mathrm{M}+1)]
$$

In practice, we found $\mathrm{M}$ equal to $3 \mathrm{~N}$ is still a manageable number.

The typical way of choosing these $\mathrm{M}$ samples is to take a rectangle patch. This brings the risk to average the pixels from different distributions, yielding a wrong estimation of the covariance matrix. A second issue is that the spatially varying fringes caused by topography and deformation lead to an underestimation of the covariance. To cope with these problems, adaptive sample selection, and a multi-resolution defringe algorithm is introduced in the following two sections.

\subsection{Adaptive Sample Selection}

The hypothesis of statistically homogenous samples is not always valid, especially under large number of samples and at boundaries of objects with different scattering mechanisms (e.g. trees, buildings). The adaptive sample selection strategy ensures the validity of the hypothesis by performing an intensity-based statistical test on the samples selection. The size and the shape of the selected sample patch can vary from pixel to pixel.

The SqueeSAR ${ }^{\mathrm{TM}}$ technique makes use of the KolmogorovSmirnov (K-S) test [5]. It is a simple and non-parametric test which measures the maximum distance between the cumulative distribution functions (CDF) of two sets of data. Different tests, such as Kullback-Leibler Divergence, Likelihood Ratio, and Anderson-Darling (A-D) test were evaluated in [6]. The A-D test was proven to provide the best performance. Different to the K-S test, it takes into account the weighted $\mathrm{L}_{2}$-norm of the difference between two CDFs.

$$
A^{2}=\frac{N}{2} \sum \frac{\left[F_{n}(x)-F_{k}(x)\right]^{2}}{F_{n, k}(x)\left[1-F_{n, k}(x)\right]}
$$

where $F_{n, k}(x)$ is the CDF of the combined data set $\{n, k\}$. The A$\mathrm{D}$ test outperforms the K-S thanks to its weighting at the tail of the difference between two CDFs, instead of considering only the maximum difference. This feature enables the A-D test to distinguish the difference between two CDFs with fluctuations of higher order [6].

The adaptive sample selection algorithm (with A-D test) and the typical rectangle method were applied on a common test site from a stack of 49 TS-X images at downtown Las Vegas. Fig. 1 compares the difference between their averaging windows, and the resulting coherence matrixes. The coherence matrices show dramatic difference. Decorrelation patterns can be clearly observed on the left (adaptive window), while the right one (rectangular window) shows generally a very high coherence $(>0.7)$, which overestimates the covariance matrix. It is caused by the presence of a number of persistent scatterers in the rectangle which happens very likely in urban areas.

\subsection{Adaptive Multi-Resolution Defringe}

Defringe, also known as phase flattening, is a necessary step to compensate the topographic phase before the covariance (or coherence) estimation. Otherwise the covariance can be underestimated due to the summing of spatially varying phase. Eq.(2) is then modified as follows:

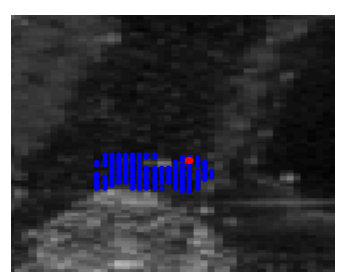

(a)

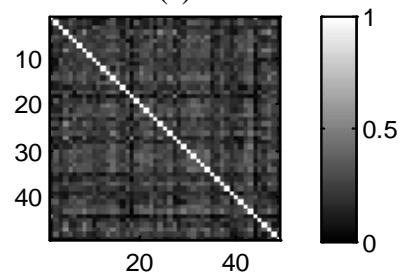

(c)

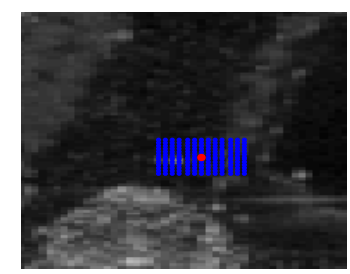

(b)

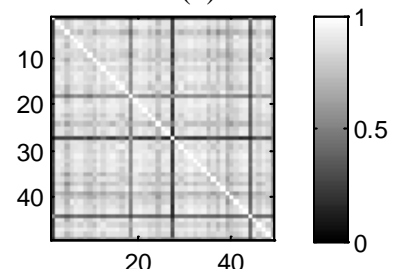

(d)
Figure 1 (a) and (b): samples selected by adaptive and rectangle sample selection strategy. The red dot in the figure is the target pixel, while those in blue are the selected pixels. (c) and (d): the coresponding covariance matrixes.

$$
\hat{c}_{n, k}=\frac{1}{\mathrm{M}} \sum_{m} i_{n}(m) i_{k}^{*}(m) \exp \left(-j \phi_{n}(m)+j \phi_{k}(m)\right)
$$

where $\phi_{n}(m)$ is the estimated topographic phase of sample $m$ in image $n$.

The typical defringe method is first to divide the interferogram into relative small patches under the assumption of a constant fringe frequency within the patch, and then to estimate the local fringe frequency by Fourier transform. However, this brings two major drawbacks: (1) the assumption of a constant frequency is not always fulfilled, especially at areas with fast varying topography, and (2) the local fringe frequency cannot be correctly estimated at areas where decorrelated noise dominant.

The proposed adaptive multi-resolution defringe algorithm overcomes these two problems by one hand removing the fringe with degressive size of local patches iteratively, and the other hand taking into account only the distributed scatterers sharing the same distribution function within a local patch. The multi-resolution feature allows it to resolve higher orders of fringe frequencies, and perform fine correction at each resolution level. The adaptive feature filters out the noise which overwrites the true local fringe spectrum.

To validate the multi-resolution defringe algorithm, it went through a test on a simulated interferogram of which certain percentage of the pixels is defined as DSs, and the rest as decorrelated noise. Circular Gaussian noises are added accordingly. The reference phase (ground truth) and simulated measurements are collocated in Fig. 2. Fig. 3 lists the input and estimated phase of each resolution level. The figure illustrates the multi-resolution strategy. Fringes within patches of sizes $100 * 100$, $50 * 5025 * 25$, and $15 * 15$ have been removed progressively. The highest resolution level $\left(15^{*} 15\right)$ was carefully chosen, because estimation error will emerge if the patch size is too small to accommodate sufficient number of DSs. Conclusion can be drawn that (1) the minimum patch size has to be chosen accordingly and depends on the number of distributed scatterers available in the 


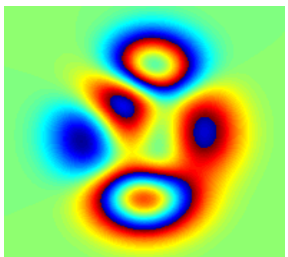

(a)

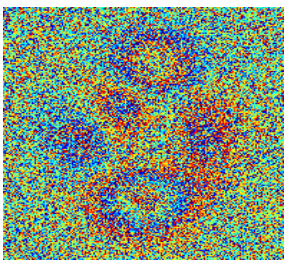

(b)
Figure. 2 (a) simulated true phase, (b) simulated measurements, generated by adding different level of noise to pre-defined DS and noise pixels.
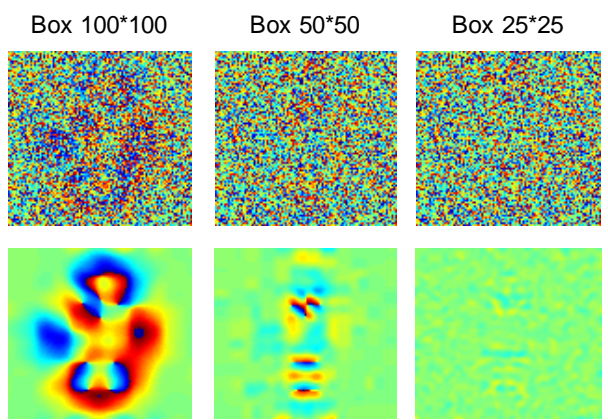

Box $10 * 10$
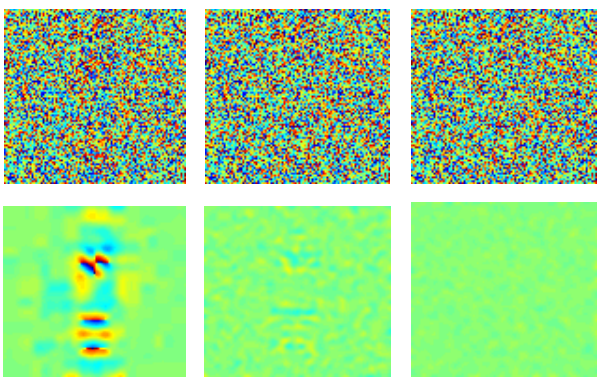

Figure. 3 Estimated fringe at each resolution level. The first row is the residual phase before each resolution level. The second row shows the estimated phase.

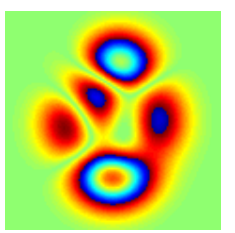

(a)

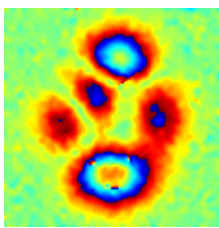

(b)

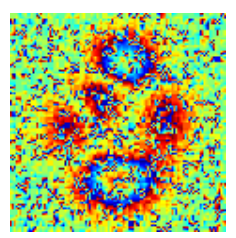

(c)
Figure. 4 (a) true fringe, (b) fringe estimated by Adaptive Multiresolution, and (c) fringe estimated by typical rectangle method

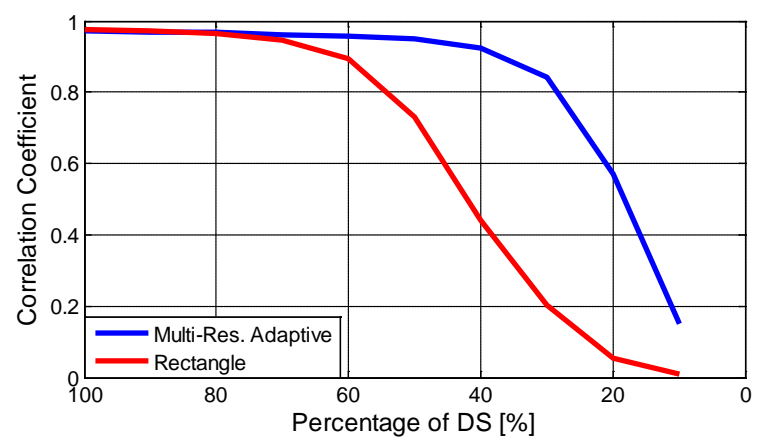

Figure. 5 Correlation coefficient of the estimated phase by adapative multi-resolution and typical rectangle method with the true phase at different percentage of DS density in the scene.

interferogram and also their coherence, and (2) patch sizes of different resolutions should be avoided to be integer multiples.

This algorithm was validated by comparing it to the typical defringe method. Both of them were applied to the same simulated interferogram used in the previous section. The findings are demonstrated in Fig. 4. The difference can be clearly observed

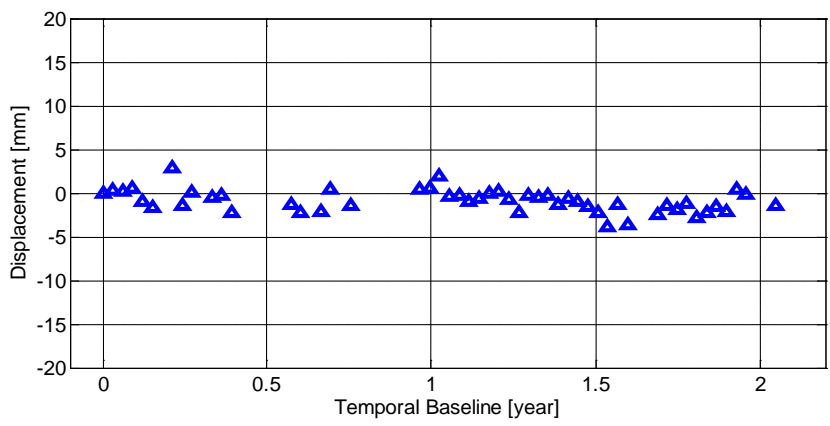

Figure. 6 Phase history of a DS. This DS shows no displacement with linear rate. Phase is converted to $\mathrm{mm}$.

between (b) and (c) that the proposed algorithm reduces the estimation error tremendously, and meanwhile, the fringe estimated by the proposed algorithm retains higher correlation with the truth than the typical method throughout a wide range of percentage of DS available in the scene, as presented in Fig. 5.

Fig. 5 also tells that the performances of the two algorithms are equivalent at situations of very high DS concentration. Both of the curves show a decreasing trend along with the decreasing percentage of the DS percentage in the scene. The number of DS is proven to be a critical criterion for both algorithms. Yet advantage of the proposed method is that it makes the best use of the information carried in the DSs.

\subsection{Phase History Retrieval}

The phase history can be retrieved from the correctly estimated covariance matrix. The phases of the first column of the covariance matrix represent the expectation value of the interferometric phase referring to the first image. This is identical to multi-looking the selected samples. Fig. 6 plots the phase history of a target DS (the red pixel in Fig. 1). This DS shows no deformation throughout the 2 years' time span.

\section{ALGORITHM TESTING}

In this section, the proposed algorithm was applied to a test site from a stack of 49 TS-X images at downtown Las Vegas. Fig. 7 shows the mean intensity of the radar return and optical image with 3D model from Google Earth. Pixels are firstly classified according to the "number of brother pixels" (pixels sharing the same distribution) of each pixel and a coarse coherence estimation (without defringe). The proposed algorithm is then performed on the DSs.

\subsection{Pixel Classification}

It is believed that most of the PSs are encompassed by very few numbers of similar pixels which share the same distribution [5], while most of the DSs will possess a great number of such "brother" pixels. Experiment is conducted on the test data. All the pixels in the test scene undergo the A-D test. The findings, in Fig. 8(a), tells that $95 \%$ of the PSs possess less than 6 brother pixels. But the numbers of brother pixels for the distributed scatterers are well over the required number of looks (in our case $L=3 \mathrm{~N} \approx 150$ ). The pixels in blue are mostly building corners, facades, and the tower. They are most likely PSs. The orange pixels are dominated 


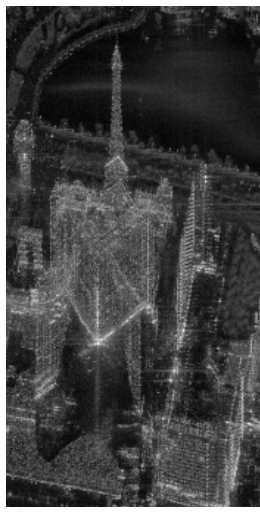

(a)

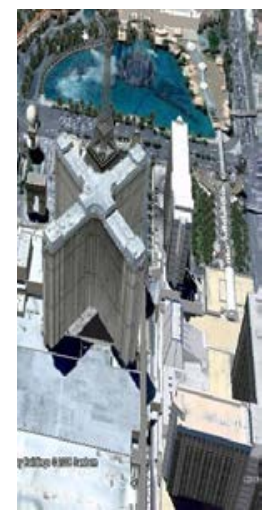

(b)
Figure. 7 (a) Mean intensity of the test site, (b) optical image with 3D model of the building from Google Earth

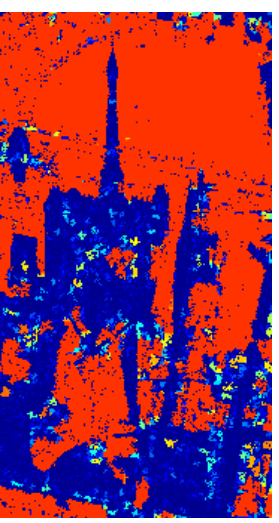

(a)
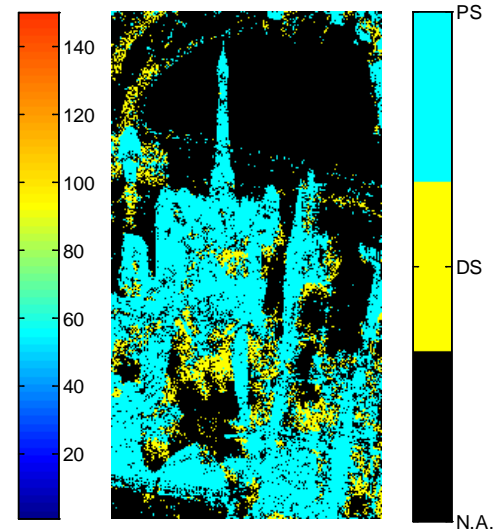

(b)
Figure. 8 (a) Number of "Brother Pixels". The colorbar represents the number, (b) Pixel Classification Map. The cyan pixels indicate the PS, the yellow the DS, and the black the unclassfied.

by surface of the lake, and the roads between the buildings. Here we make use a coarse coherence estimation to distinguish DS and decorrelated noise. The final classification map is shown in Fig. 8(b).

\subsection{Estimated Parameter}

Fig.9 shows the estimated elevation of PSs (by standard PS technique), and DSs (by the proposed algorithm). An increase in the information coverage over the city can be observed. The increment can be more significant in case of rural and mountainous region where DSs are widely distributed.

\section{CONCLUSION}

This article presents the first demonstration of parameter estimation by MLE at distributed scatterers using very high resolution TS-X spotlight data. Particular focus is put on the critical problem of accurate covariance matrix estimation. The proposed algorithm introduces an adaptive sample selection and multi-resolution defringe methods to cope with this issue. The adaptive sample selection method is proven to be an essential procedure in the covariance matrix estimation. The A-D test

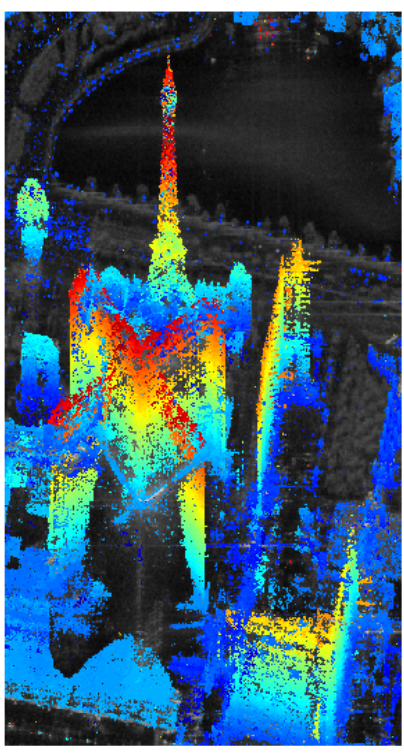

(a)

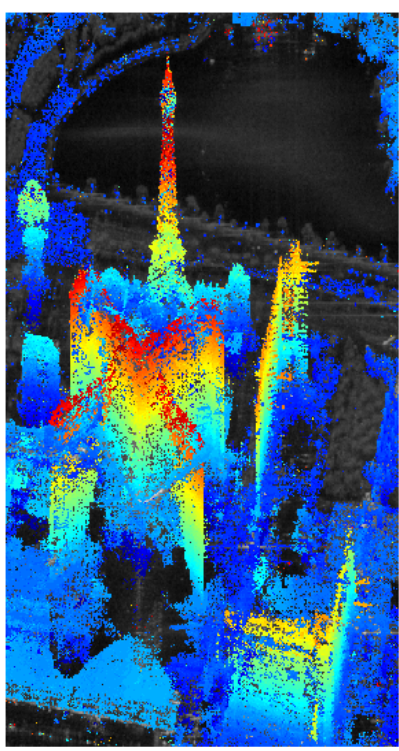

(b)

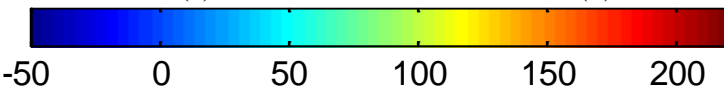

Figure 9 (a) Estimated elevation at PSs, (b) Estimated elevation at PSs and DSs. The eleveation is in meters and color coded.

performs well in distinguishing targets of different scattering characteristics. The proposed adaptive multi-resolution defringe algorithm outperforms the typical method over a wide range of DS densities. Through the experiment of all the procedures of the proposed algorithm, this paper also provides a simple way of pixel classification by A-D statistical test and coarse coherence estimation for high resolution TS-X data.

The findings reveal its potential at places rich of distributed scatterers, such as rural areas and volcanoes. Applications such as natural hazard monitoring are of great attraction. It is also interesting to investigate the capability of MLE under the condition of small number of acquisitions.

\section{REFERENCES}

[1] Bert M. Kampes, Radar Interferometry: Persistent Scatterers Technique. Dordrecht, The Netherlands: Springer, 2006.

[2] R.Bamler, P.Hartl, "Synthetic aperture radar interferometry", Inverse Problem, Vol. 14, 1998, pp. R1-R54

[3] M. Seymour, I. Cumming, "Maximum Likelihood Estimation For SAR Interferometry", In: Proceedings of IGASS Conference, 1994

[4] F. De Zan, "Optimizing SAR Interferometry for Decorrelating Scatterers”, Doctoral dissertation. Milan, Italy, 2008.

[5] A. Ferretti, A. Fumagalli, F. Novali, C. Prati, F. Rocca, A. Rucci, "Expoitation of Coherence Matrixes in Multi-temporal SAR Datasets: the SqueeSAR approch”, In: Proceedings of IGARSS Conference, 2010.

[6] A. Parizzi, R. Brcic, "Adaptive InSAR Stacks Multi-looking Exploiting Amplitudes Statistics. A Comparison between Different Techniques and Practical Results”, IEEE GRSL, Vol. PP, Issue. 99, 2010, pp. 441 - 445

[7] I.S. Reed, J.D. Mallett, L.E. Brennan, "Rapid Convergence Rate in ADAPTIVE Arrays”, IEEE Trans. AES-10, 1974, pp. 853-863. 